\title{
Challenges Faced By the Indian Transportation Sector in Implementing New Age Technologies
}

\author{
Sandip Tripathy 1 \\ ${ }^{1}$ Symbiosis Institute of Operations Management, Nashik, Symbiosis International (Deemed University), Pune, India
}

\begin{abstract}
This research paper in its entirety tries to articulate the various types of challenges faced by the Indian Transportation Sector in implementing new age technologies for better control and visibility throughout the entire Supply Chain. It mostly focuses on the road and rail mode of transportation, though briefly it also touch-bases upon the water and air modes. Indian railways and roads position themselves as the third and second largest network respectively worldwide. In addition to a set of questionnaires circulated among individuals working in the logistics domain to capture their point of view, a thorough literature review of the previous works in this field was done to identify the causes - both external and internal to an organization, which affects implementation of digital solutions to present transportation problems. In this paper, we discuss along with others, two of the greatest challenges posed to the digital transformation - firstly, adamant attitude of traditional players operating in this space, who have for long, functioned through traditional processes and legacy systems and secondly, the complexity of the road and rail network running through the length and breadth of the country. Though the average time taken by enterprises to digitize their transportation business has reduced tremendously due to need of remote operations in these unprecedented times during COVID19 , but still there exist some of the challenges which need to be addressed. Some of the major drivers of identifying these challenges are the need to - reduce lead times by setting up a platform/control tower which can automate the management of transportation with minimized touch points between organizations and their customers, need to reduce risk of manipulated data by minimizing human intervention and need to increase customer satisfaction by incorporating functionalities like real-time tracking, automated exception handling and other. The challenges which were identified in this study can be further analysed to improve on it and conceptualize a framework for transforming Indian logistics (majorly the transportation space). This paper is written from the point of view of current scenario of Indian transportation industry.
\end{abstract}

Article Received: 10 August 2020, Revised: 25 October 2020, Accepted: 18 November 2020

\section{Introduction}

Transportation plays a vital role in the Supply Chain of any organization. It forms a crucial link between the various stakeholders involved at various stages. It is this phase when the strategy is actually put to action and physical movement of goods / products and exchange of ownership from the source to the end consumer takes place.

World Economic forum 2016 report indicates that the logistics space will position itself at a value of more than 1 trillion dollars. According to a study by Novonous - a marketing research firm, India spends approximately $15 \%$ of its Gross Domestic Product (GDP) on transportation as against an average of $8-9 \%$ by other developing countries (Sharma and George, 2018). From an Indian perspective, transportation mostly comprises of roadways and railways with a vast network of both running throughout the country (Patel, 2017) with road network covering nearly 4.4 million kilometres and rail network stretching along a length of nearly 1.2 lakh kilometres (as per recent data from Ministry of Road Transport and Highways \& Indian Railways). Road transport contributes heavily in terms of carrying passenger traffic with its share being more than $70 \%$, according to reports from World Bank. It is also a heavily fragmented sector consisting of several players with most of them operating in a traditional way manually or through legacy systems. Due to this fragmented nature, benefits of economies of scale cannot be reaped (Patel, 2017). Though transportation in India also consists of the water (inland waterways) and air mode, with water mode being the cheapest (Potluri and Tejaswi, 2018), this study is mostly focussed on road and rail modes. Transportation costs forms a major chunk of supply chain costs (Chandra and Jain, 2007) which needs to be optimized and also within transportation cost, fuel cost forms the crucial component. Transportation cost is the area of concern for most of the Indian Supply chains and they always try to optimize it (Chandra and Jain, 2007). The transportation sector is going through a transformational phase or we can say that it is experiencing digital disruption. This paradigm shift is more pronounced due to the ongoing COVID-19 which has suddenly brought to attention the need to 
digitize the supply chain in general and logistics space in particular. Now that most of the work is done remotely, there is an increasing need for automated processes which can be managed remotely. From an Indian perspective, large chunk of the trucks / vehicles that ply on the National Highways or the Expressways carry more than their designated capacity and either travel more than the allowable limit which then leads to exertion and health degradation for the vehicle staff and driver and increased fuel consumption (Yan and Crookes, 2010), which in turn causes an increase in fuel cost which is the largest contributor to transportation costs. There are also instances when these vehicles run for less than 300 kilometres which is less than one-third of the average of the distance vehicles run in developed countries (Chandra and Jain, 2007). Therefore, both the scenarios are inefficient and needs to be optimized. Also, transportation has been heavily contributing to the carbon emission due to multifold increase in urbanization and industrialization due to which there is an increase in congestion (Potluri and Tejaswi, 2018). The transportation space consists of both traditional and tech-enabled players both of which needs to come together in a single platform. For achieving this, we need to train the traditional players (transporters), the drivers and other ground staff and hand hold them till the point where they can operate in the digital space independently. Due to technological advancement, expectation of customers (particularly those who are using some kind of logistics / transportation services) has increased and they want visibility which is similar to what ecommerce giants like Amazon and Flipkart offers. Therefore, the companies have realised that it order to maintain their customer satisfaction indices, they need to transform the transportation business. Companies also view digital transformation of the transportation space as a strategic move (F Wei et al., 2019). Some studies propose the use of blockchain in the transportation sector for facilitating ITS (Intelligent Transport System) where a client-server framework is developed which monitors and stores the information of a car in transit and finally this information is used to develop the ITS (Hirtan, L. A., \& Dobre, C. , 2018).

To understand the challenges posed to implementation of technology in transportation, first we have to understand how a digitized transportation ecosystem looks like. Vehicles embedded with GPS devices, control tower for managing the end to end logistics, electronically capturing documents like proof of delivery, dashboards showing performance and analytics reports are some of the examples of functionalities in a digital transportation ecosystem. Technology helps in various ways in this leg such as route optimization, automated alerting to stakeholders during any exception, alerting to customers during gate-out and gate-in based on geofencing, realtime tracking of shipments and many more. Challenges that are posed to the technological advancement at this leg of logistics can be identified and analysed at all three levels namely Strategic, Tactical and Operational level (F Wei et al., 2019). Though the most profound influence is experienced at the operational level (F Wei et al., 2019), yet other two levels (strategic \& tactical) also experience it to some extent. Since we see that effects are more prominent at the operational level, it becomes even more important to address the issues faced by ground staff and drivers ( $F$ Wei et al., 2019). A sector which provides livelihood for more than 20 million people (Yadav et al., 2020) needs to advance and cope up with the customer expectations so as to remain competitive. Combination of road and rail modes into an intermodal system is efficient in terms of both costs and environmental sustainability (Kumar and Anbanandam, 2019). Therefore the focus should be on implementing technology based solutions to intermodal system rather than building individual solutions for road and rail. Digitization is not completely alien to the Indian transportation and there have been some projects undertaken by GOI which involves use of technology as an enabler to provide better services, increase control and efficiently manage the entire game. But, these projects face challenges in terms of implementation even after proper planning at several hierarchical levels is done. Therefore, the field of transportation remains an area of potential improvement in terms of addressing the challenges of implementation and optimizing the operations. Several methods have been used to optimize the network traffic, with one such method being the $\mathrm{A}^{*}$ algorithm which uses $\mathrm{R}$ tree methodology along with KNearest Neighbours algorithm and google maps to find the least congested route between a source and a destination (Soe, N. C. \& Thein, T. L. L. , 
2018). Distribution network optimization can be performed using 2E-LRP (2 echelon-location routing problem) model where the network optimization is done using the concepts of Operations Research, particularly Integer Linear Programming. Several nodes are setup with capacity and supply constraints and then Mixed Integer Linear Programming is used to reach to the optimal route (Wang, Y., Tian, X., \& Liu, D. (2017). Tanaino, I., Yugrina, O., \& Zharikova, L. (2019) emphasizes the importance of transportation in business and focusses on the multimodal systems and use Just-In-Time concept to optimize shared transport and freight operations.

\section{Literature Review}

Here we look at some of the previous works relevant to transportation and logistics space to set the context and understand the challenges related to transportation

Patel (2017) sheds lights on some of the challenges faced by the Indian transportation industry in terms of poor infrastructure, lack of proper training for ground personnel, drivers and other staff and increased congestion due to surge in vehicles plying on the road leading to saturation of transportation networks. Congestion leads to slowing down of speed of the vehicles which means more emissions into the atmosphere as studies from Centre of Science and Environment shows there is a negative correlation between the speed of vehicle and the quantity of emission from the vehicle Therefore improved connectivity not only saves times and cost, but it also helps to contribute to environmental sustainability.

Cichosz et al. (2020) focuses on understanding barriers and key success factors pertaining to adoption of digital technologies in logistics space. This paper segregates organizations into four quadrants depending on the degree of intensity of transformation management and digitalisation. It says that the leaders of organization face challenges in terms of demand for digital adoption from customers and other stakeholders. Therefore companies have introduced several measures one of them being the introducing roles of Chief Digital Officer (CDO) to drive digital transformation processes smoothly and efficiently. Barriers to digitalisation such as difficulties in automating complex processes, lack of skilled workforce and organizational culture were identified. Also, some of the important factors for successful transformation were identified as efficient leadership which could drive the change seamlessly, employee engagement and alignment of strategies and business processes.

Ramji \& Venugopal, (2019) in their paper estimate that the size of mobility industry of India is more than 600 million USD and will continue to grow exponentially. Also, Mobility as a Service is seen as a disruptive model focussing on shared usage instead of ownership. Role of government and private organization is crucial in creating a sustainable mobility framework. Shared usage is beneficial from the perspective of environment focussing on minimizing Carbon Dioxide emission.

Queiroz et al. (2019) in their research defines digital supply chain as a connected network of players with effective communication and synchronization between them to make the network agile and realize better outcomes in terms of service level. The paper further goes on to discuss the capabilities of a decentralized digital supply chain. Digital supply chain capabilities are defined as technological resources that transform the operational activities and improve visibility and help focus on collaboration rather than working in silos. Queiroz et al. (2019) identified several enablers and capabilities to supply chain digitalization with the enablers being big data analytics, blockchain technology, artificial intelligence, cyber physical systems, IoT and cloud computing while the capabilities being supplier and customer integration, compliance and policies, warehouse and transportation capabilities and smart production

According to Chandra and Jain (2007), due to surge in demand, the transportation infrastructure has been stretched to perform over its limits and this has become the reason for innovations in the transportation space. Moreover, due to factors like cost efficiency and flexibility, there is an inclination to prioritize the digitizing of road transportation. There have been numerous attempts by government bodies such as the National Highway Authority of India (NHAI) to automate certain processes via the implementation of intelligent transport system, but complete integration still remains a challenge due to several factors, one of them being lack of trust and awareness among the players. These shortcomings calls for building and maintaining efficient 
knowledge management infrastructure which helps the logistics firms (or, specifically, players operating in the transportation space) remain competitive (Yadav et al., 2020). Knowledge management essentially refers to the technical and IP knowledge that is accessible to the employees of an organization. Knowledge management systems have already proved to be strategic for many supply chains and continue to grow with them.

Kumar and Anbanandam (2019) discuss largely about the intermodal system of transportation. Intermodal transportation refers to the combined use of road and railways at the large to facilitate movement. Further, it is discussed by them that this combined approach has greater cost efficiency, improved speed and better security as compared to the unimodal system. Despite having several advantages of using an intermodal system, still there are challenges faced in terms of implementation largely because there is lack of real-time data availability. Moreover, intermodal system proves to be cost efficient in cases of longhaul distances and large volumes only but when it comes to shorter distances and less volume, the cost benefit cannot be reaped (Kumar and Anbanandam, 2019). According to 2016 report of GOI, the industry faces acute problems related to network congestion, increased emissions and poor infrastructure and the intermodal system can help minimize the effects of the these challenges. Also the Indian Government plans to achieve equal distribution between roads and railways by 2002 , according to reports from NTDPC.

F. Wei et al. (2019) states that digitization has become one of the top 15 identified trends in the logistics space and that more than three-fourths of the companies view digitization as a strategic move. Further, their study shows that the analysis of digitization is taken to all the aspects that affect the triple bottom line of an organization namely social, ecological and economical. The purpose of digitization is to reap the maximum benefits by using the minimum resources and also minimizing emissions by optimization. There exists some challenges to digitization such as IT incompetency and data security issues due to a large portion of the key employees having operated in a traditional way for long (F. Wei et al., 2019).

Raina et al. (2019) mentions about the increase in urbanization in the previous decade which saw a rise from $28 \%$ to nearly $31 \%$ and also the fact that only the transportation industry accounts for nearly one-thirds of the world's energy. As we know that increased urbanization has put pressure on the existing transportation infrastructure causing saturation of the network, therefore optimized solutions are needed and this will minimize the strain on the existing resources and help restore and maintain the ecological balance. Resilience is an important aspect to be taken into consideration while framing strategies for the supply chains.

Sharma and George (2018) discuss about resilience in truckload transportation industry in terms of two broad topics - firstly, the ability to restore to normal operations quickly after disruptions and secondly, the ability to contain and avoid the disruptions without being affected much. Some of the features of resilience as put forward by Sharma and George (2018) are preparedness and operational flexibility. Effective communication and coordination within the players are the two major drivers of resilience.

Mishra (2020) suggests developing an intelligent system comprising of integrated components using state-of-the-art technologies like IoT, cloud computing and others that would help minimize the road accidents. Further in his study, it is articulated that most of the casualties in road accidents occur due to the lack of knowledge/information on what steps should be taken to save a life and this situation can arise due to multiple reasons, some of them being relatively new geography, little or no awareness about vehicle conditions and so on. While there is no control on when an accident would happen, some of the effects of accidents like majorly wounded, deaths etc. can be mitigated by the help of intelligent systems installed at the various nodes/checkpoints.

Study relating to the characteristics of truck drivers to understand the traits and behaviour of the drivers for sustainable transportation carried out by Dubey and Gunasekaran (2016) suggests that satisfaction is one of the important drivers of motivation to remain focussed in professions like transportation where there are instances of longhaul movement of more than two or three days which often leads to exertion and frustration. The study focuses on several types of hardships and challenges faced by the drivers and propose methods like training and mentoring to improve the conditions. 


\section{Research Methodology}

The aim of this paper is to identify the challenges posed to the implementation of new age technologies in the transportation space. As an attempt to identify gaps and explore relationships between the various stakeholders involved in transportation, a two-way approach was followed. Firstly, a comprehensive literature study was done for 14 research papers (all of which published in Scopus / Web of Science indexed journals) pertaining to transportation domain to understand what work has been done till date in this field and the existing pain points in transportation as mentioned in those papers. The research papers were analysed to identify the current situation prevailing when a particular paper was written, methodology developed to overcome or improve on the current scenario and further implications of the implementation. Based on these insights from the papers and responses from the survey, relevant findings were identified to be the key issues in technology implementation. The research papers were selected by using combinations of keywords mentioned below:

- Transportation

- Challenges

- Digital Disruption

- Logistics

- Digitization

- Smart Integration

The research papers that were studied were accessed from the Scopus database. Papers were filtered as "Open Access" within the Access type option and as "Business, Management and Accounting" within the Subject area option. Within these types, the ones which were relevant to transportation and digitization were selected to be analysed and studied.

Secondly, a set of questions were floated to Supply Chain and Logistics professionals. The questions were formed according to the analysis done on the studied research papers by taking into account the pain points as mentioned in the research papers. The questionnaire consisted of 9 multiple choice questions and 3 questions measured on Likert scale of 1 to 5 . The questions were floated to 350 working professionals across various organizations through email and LinkedIn and responses of 200 professionals were received. The survey responses were recorded from $13^{\text {th }}$ August, 2020 to 24th August, 2020. Responses to multiple choice questions were visualized using doughnut charts in Ms-Excel. Likert scale responses were analysed by using Chi-square functionality in the data analysis feature of MsExcel. Chi-square test was done to calculate the probability of randomness in the responses.

\section{Findings \& Analysis}

We take a look at the Likert scale responses and analyse them to calculate the probability of randomness. Doughnut charts are used to visualize the responses of the survey. Based on the responses of multiple choice questions from the survey, key reasons that identified themselves as hindrance to technological implementation are discussed below. 


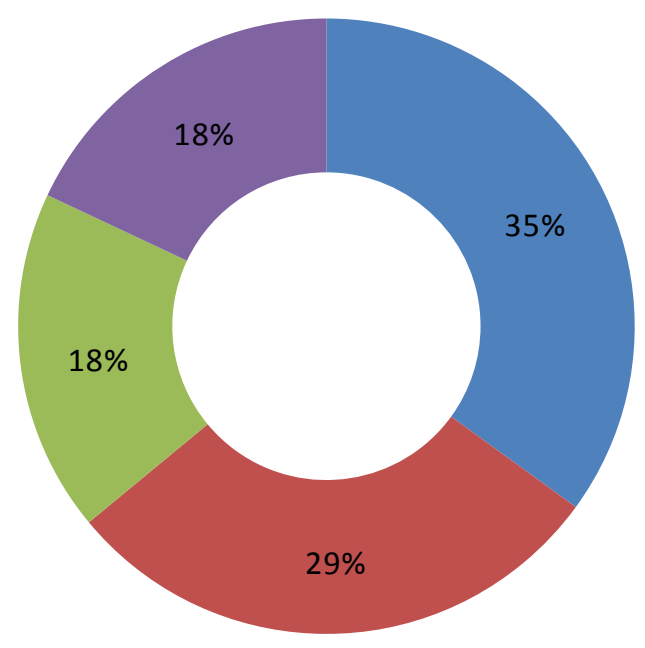

Traditional and Legacy players not willing to embrace technology and change

Complex network of Roads and Railways - making it difficult to conceptualize a solution

- Fragmented nature of the Industry

Uncertainty of future operations due to Covid-19

Figure 1 - breakup of 200 responses showing key challenges to implementation of technology in transportation

Players in the transportation space who have for long operated using legacy systems are not willing to adopt new technologies. $35 \%$ of respondents are of the view that this is most prominent challenge in implementation (Figure 1). They are resisting changing to a new ecosystem of technology driven operations. Even if implementation is done, profits and other benefits cannot be reaped without the ground players accepting the transformation. Moreover, the very purpose of digitization which is to increase visibility and control in addition to saving time can only be served when these players (mostly transporters or logistics service providers) are ready to embrace technology. Since more than $68 \%$ of the respondents surveyed use road mode on a regular basis, we take an example of an organization which wants to implement TMS (transportation management system) for its inbound and outbound logistics via road mode, then it is important that the transporters and drivers are trained properly. If there is a track and trace functionality in the TMS, then the driver needs to know how to give consent digitally for tracking the vehicle he is driving. As we know that most of the transporters are still using the traditional methods, it is evident that it will require some time to train them and bring them onboard, but the very first thing should be persuade them to agree to use technology and receive training because no matter how much training and hand-holding is done, if the transporter / logistic service provider is not willing to change ways, the entire process will remain inefficient due to less visibility and control.

India has a vast and complex network of roads and railways that make it difficult to conceptualize a technology based solution for optimization. Implementing digital solutions for long-haul movements is difficult (Figure 1). To illustrate this, we take an example of a long-haul movement in road transportation where we implement control tower technology for track and trace functionality as a part of TMS. Often it is seen that the vehicle changes during these type of movements and these changes causes the control tower to switch between different mode/device accordingly and due to this switching, visibility and control become somewhat limited. Railway network of India is one of the largest in India and covers some of the difficult terrains such as the Pir Panjal mountain range in Jammu \& Kashmir and the Bogibeel rail-road dual bridge over Brahmaputra River in Assam. Due to such geographies, it becomes difficult to implement technology uniformly throughout the network and even if we succeed in implementing, benefits cannot be fully reaped.

The fragmented nature of the transportation industry as evident from the fact that on an average, a transporter owns not more than 5-7 vehicles, contributes to the issues faced in its implementation of technology in this space (Figure 1). As far as road transportation is concerned, there are a large number of players operating in this space, some of them are well- 
equipped and tech-enabled and others operate traditionally and therefore integrating with each one of them to onboard on a common digital platform is a cumbersome process. This is also justified by the survey where more than $52 \%$ of the respondents are of the view that the complexity level is moderate to difficult when it comes to integrate all type of players in a single digital platform. Moreover, different transporters differ in their processes and to map them to a single consolidated workflow requires extensive planning and is even more difficult to achieve. As a low literacy level prevails across this industry, particularly when we narrow down to drivers, support staff and the likes, it becomes even more difficult to consolidate with all of them for all the players. When asked to the respondents whether political conditions will affect this transformation, more than $75 \%$ answered positively. Political conditions prevailing in an area can drive technological advancements in transportations and help in achieving the desired reduction in cost and time. Another major issue of implementation of technology as evident from the responses are the concerns related to data security. Digitization captures tremendous amount of data and some of it being very crucial to business; therefore there have a lot of debates in the past relating to the breach of data security. Uncertainty of future operations also poses a challenge to implementation of technology in the transportation space. More than $55 \%$ of the respondents believe that it is moderate to difficult when it comes to implementing these solutions while not being present physically at the warehouse or distribution centre/depot but rather working remotely.

More than $70 \%$ of the respondents are of the view that inadequate and saturated infrastructure is the main reason for the delay in run time of railways. Lack of infrastructure poses a challenge to the implementation of digital solutions as it makes the planning difficult due to non-uniformity. When it comes to road transport, more than $55 \%$ of the respondents say that the present conditions of national highways in India is moderate. More than $35 \%$ of the respondents say that according to the current conditions, it would take between 12 to 18 months for full implementation of technological solutions (Figure 2). More than 55\% of the respondents say that introducing technological advancements will help to reduce transportation costs. Introducing technology will minimize human intervention and thus will reduce manipulation and increase efficiency which eventually minimizes the cost and saves time.

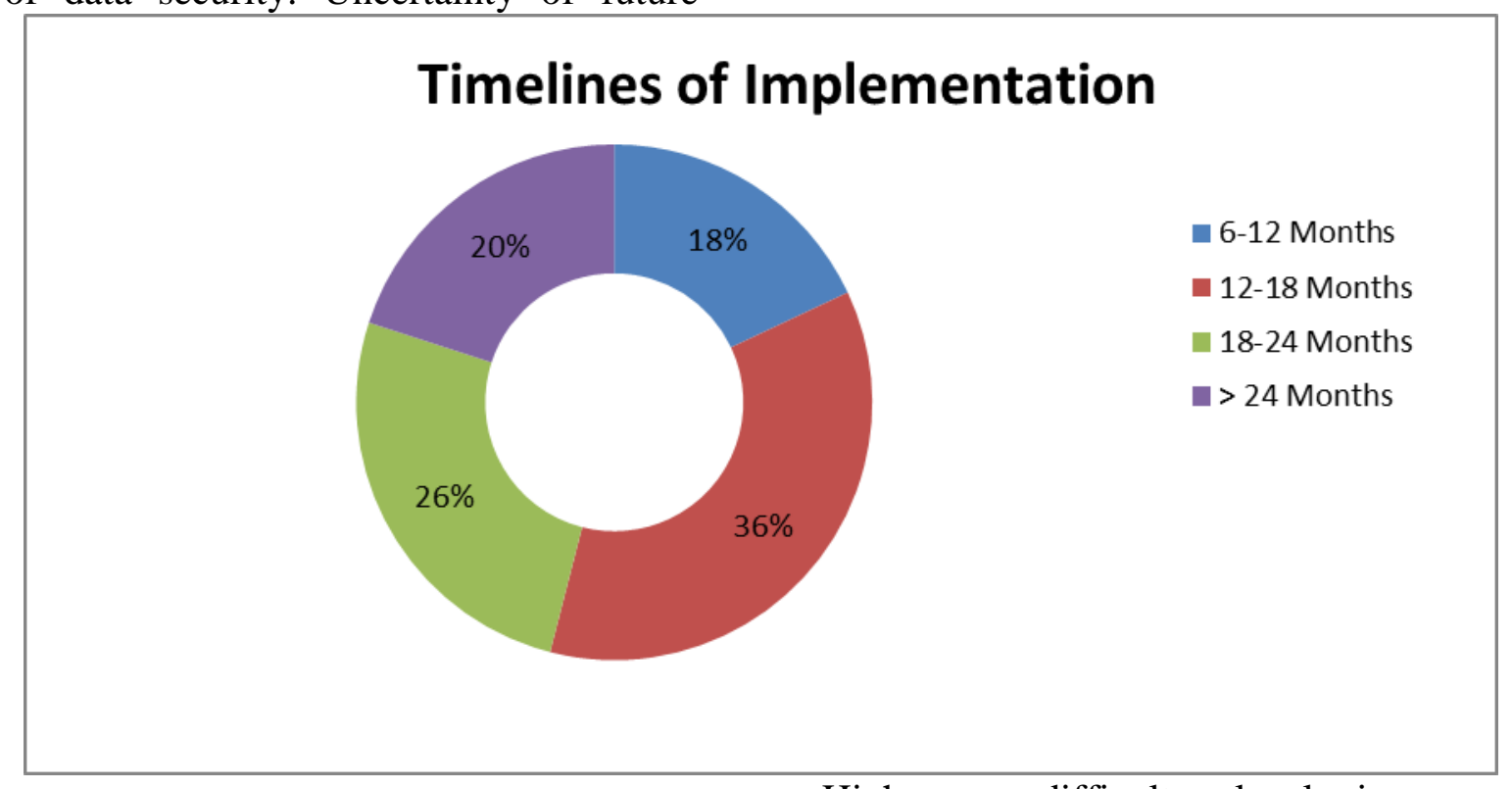

Figure 2 - breakup of 200 responses showing how much time it would take to implement technology The chi square test done on the Likert scale responses for questions regarding the present operating conditions of Indian National
Highways, difficulty level in consolidating different players (both traditional and techenabled) into a single digital platform and level of difficulty encountered in remote implementation was done to calculate the probabilities of getting the results by randomly answering the questions. 
The chi square value for these Likert scale responses were very small (all the three values being smaller than 0.0001). Low probability values mean lower chances of getting these response values just by fluke. Therefore it suggests that these values are rational enough to be considered as legitimate response values.

\section{Conclusion \& Limitations}

This paper titled identifies the problems in using digital and new age technologies in the transportation space. During these times, all the organizations are pushing hard to redesign their supply chains to make them resilient and digitizing their logistics is an integral part of this effort. Therefore, identifying the challenges in implementation of technology based solutions is imperative in driving the change in the global supply chain. As we will move towards an uncertain after-Covid-19 world, it is expected that there will be a paradigm shift in the way of working. There are examples being set up by many companies who have already switched to remote operations. In the days to come, technology will be the key to competitive advantage so it is important to identify which type of technology should be used in various business scenarios. When an organization's higher management comes up with a strategy to implement technology in this space, the concerned teams will be confronted with challenges and after that organizations have to involve their stakeholders and other players in the supply chain to decide on the course of implementation while simultaneously coming up with solutions to the problems. This paper talks about the main challenges faced in terms in unwillingness to adapt technological change, complexity of infrastructure and transportation network and fragmented nature of the transportation sector in India where there are a large number of players. While the current restrictions are inevitable, organizations should find out ways to drive transformation in transportation space by continuing to work remotely and this can be done only by proper use of technology. Identifying the challenges before the business cases are defined will provide managers with an opportunity to conceptualize solutions to the various problems discussed earlier in this paper. As Supply Chains tend to become more resilient in the post covid-19 world, visibility and control will become imperative and to achieve it, technology will play a major role and for technology to be implemented efficiently, we need to counter the challenges pertaining to these technologies

\section{Limitations}

It is believed in the Supply Chain world that to get to know about the operational details and processes wisely, one has to visit various supply chain nodes such as warehouses, distribution centres, logistics centres and the likes. Given the limitation of working from home in these unprecedented times, all the research that was done was with the help of internet by analysing several research papers and surveying supply chain and logistics professionals. One of the major limitations of this paper was the inability to physically go transport hubs or to transporters to study the on-ground challenges faced by them. It was highly unlikely during current scenarios to get hold of transporters to communicate or get hold of them for a meeting or two. Another limitation is that majority of the paper revolves around road and rail modes of transport as these are majority from the Indian perspective (justified by the fact that more than $90 \%$ of the respondents use road and rail modes). Air and Sea modes are also prevalent in India but these modes are already digitized, atleast more than road and rail modes. Therefore more challenges can be identified by actually visiting the transporters / logistics service providers and understanding the on ground challenges and this part of the work remains as the future scope of this paper.

\section{References}

[1] Authors, F. (2017). Benchmarking: An International Journal.

[2] Chandra, P. (2014). The Logistics Sector in India : Overview and Challenges The Logistics Sector in India : Overview and Challenges Pankaj Chandra Nimit Jain W . P . No . 2007-03-07 The main objective of the working paper series of the IIMA is to help faculty members, Resea (Issue January 2007). https://doi.org/10.1142/9789812814661

[3] Cichosz, M., Wallenburg, C. M., \& Knemeyer, A. M. (2020). Digital transformation at logistics service providers: barriers, success factors and leading practices. International Journal of Logistics 
Management, 31(2), 209-238. https://doi.org/10.1108/IJLM-08-2019-0229

[4] Dubey, R., \& Gunasekaran, A. (2015). The role of truck driver on sustainable transportation and logistics. Industrial and Commercial Training, 47(3), 127-134. https://doi.org/10.1108/ICT-08-2014-0053

[5] Hirtan, L. A., \& Dobre, C. (2018). Blockchain privacy-preservation in intelligent transportation systems. Proceedings - 21st IEEE International Conference on Computational Science and Engineering, CSE 2018, 177-184. https://doi.org/10.1109/CSE.2018.00032

[6] Kumar, A., \& Anbanandam, R. (2020a). A Flexible Policy Framework for Analysing Multimodal Freight Transportation System in India: SAP-LAP and Efficient IRP Method. Global Journal of Flexible Systems Management, 21(1), 35-52. https://doi.org/10.1007/s40171-019-00226-5

[7] Kumar, A., \& Anbanandam, R. (2020b). Analyzing interrelationships and prioritising the factors influencing sustainable intermodal freight transport system: A greyDANP approach. Journal of Cleaner Production, 252, 119769. https://doi.org/10.1016/j.jclepro.2019.11976 9

[8] Mishra, K. N. (2020). A novel integration of smart vehicles and secure clouds for supervising vehicle accidents on roads/highways. Sadhana - Academy Proceedings in Engineering Sciences, 45(1). https://doi.org/10.1007/s12046-020-1320-9

[9] Patel, D. M. (2017). Analysis of Indian Transport System. International Journal of Trend in Scientific Research and Development, Volume-1(Issue-3), 27-30. https://doi.org/10.31142/ijtsrd74

[10] Potluri, R. M., \& Tejaswi, S. P. (2018). Challenges of transport sector in India: A dyadic perspective. Journal of Asian Finance, Economics and Business, 5(3), 95102. https://doi.org/10.13106/jafeb.2018.vol5.no 3.95
[11] Queiroz, M. M., Pereira, S. C. F., Telles, R., \& Machado, M. C. (2019). Industry 4.0 and digital supply chain capabilities: A framework for understanding digitalisation challenges and opportunities. Benchmarking. https://doi.org/10.1108/BIJ12-2018-0435

[12] Raina, S., Madapur, B., \& Kollarath, R. M. (2019). Green transportations systems - a step towards sustainable cities. International Journal of Recent Technology and Engineering, 8(2 Special Issue 3), 924-926. https://doi.org/10.35940/ijrte.B1174.0782S3 19

[13] Ramji, A., \& Venugopal, S. (2019). Creating a Sustainable Mobility Ecosystem in India: Vision 2030. 2019 IEEE Transportation Electrification Conference, ITEC-India 2019. https://doi.org/10.1109/ITECIndia48457.2019.ITECIndia2019-256

[14] Soe, N. C., \& Thein, T. L. L. (2018). An Efficient method of Optimal Routing System for Transportation (Yangon). ISCIT 2018 - 18th International Symposium on Communication and Information Technology, Iscit, 180-185. https://doi.org/10.1109/ISCIT.2018.858794 3

[15] Tanaino, I., Yugrina, O., \& Zharikova, L. (2019). Routing of freight transportation in logistics of agriculture. IOP Conference Series: Earth and Environmental Science, 403(1). https://doi.org/10.1088/17551315/403/1/012192

[16] Wang, Y., Tian, X., \& Liu, D. (2017). Optimization of urban multi-level logistics distribution network based on the perspective of low carbon. Proceedings 2017 Chinese Automation Congress, CAC 2017, 2017-January, 4896-4900. https://doi.org/10.1109/CAC.2017.8243646

[17] Wei, F., Alias, C., \& Noche, B. (2019). Applications of digital technologies in sustainable logistics and supply chain management. Innovative Logistics Services and Sustainable Lifestyles: Interdependencies, Transformation Strategies and Decision Making, 235-263. 
https://doi.org/10.1007/978-3-319-98467-

4_11

[18] Xie, C. (2018). Research on Logistics Facilities Location Based on GIS. Proceedings - 2nd International Conference on Data Science and Business Analytics, ICDSBA 2018, 358-361. https://doi.org/10.1109/ICDSBA.2018.0007 4

[19] Yadav, D. K., Pant, M., \& Seth, N. (2020). Analysing enablers of knowledge management in improving logistics capabilities of Indian organisations: a TISM approach. Journal of Knowledge Management. https://doi.org/10.1108/JKM10-2019-0535

[20] Yan, X., \& Crookes, R. J. (2010). Energy demand and emissions from road transportation vehicles in China. Progress in Energy and Combustion Science, 36(6), 651-676.

https://doi.org/10.1016/j.pecs.2010.02.003 